\title{
On Sample-Path Staleness in Lazy Data Replication
}

\author{
Xiaoyong Li, Daren B.H. Cline, and Dmitri Loguinov
}

\begin{abstract}
We analyze synchronization issues arising between two stochastic point processes, one of which models data churn at an information source and the other periodic downloads from its replica (e.g., search engine, web cache, distributed database). Due to lazy (pull-based) synchronization, the replica experiences recurrent staleness, which translates into some form of penalty stemming from its reduced ability to perform consistent computation and/or provide up-to-date responses to customer requests. We model this system under non-Poisson update/refresh processes and obtain sample-path averages of various metrics of staleness cost, generalizing previous results and exposing novel problems in this field.
\end{abstract}

\section{INTRODUCTION}

With the massive growth of the Internet and deployment of large-scale distributed applications, mankind faces new challenges in acquiring, processing, and maintaining vast amounts of data. In response to this flood of information, companies deploy cloud-based solutions designed to provide replicated and distributed support to the skyrocketing storage and processing demand of their users.

One interestingroblem in these applications is the highlydynamic nature of content, especially when perfect synchronization of sources, replicas, intermediate caches, and various computation is impossible. In fact, many large-scale distributed systems (e.g., airline reservations, online banking, web search engines, social networks) operate under constant data churn and may never see consistent snapshots of the entire network. As a result, these applications may hold and/or manipulate a mixture of objects that existed at the source at different times $t$ in the past. This leads to questions about staleness, synchronization costs, and techniques for deciding optimal refresh policies.

In traditional databases, the source opens outbound communication with the replicas whenever it detects important information changes. This enables push-based operation that actively expires stale content and broadcasts notifications into the system. Even under multi-hop replication, staleness lags in these systems are described by simple models that can be reduced to convolutions of single-hop notification delays.n other cases, however, scalability and administrative autonomy require that sources operate independently and provide information only based on explicit request, especially when they are unable to track all of their replicas or adopt modifications to existing protocols

This pull-based replication (also called optimistic or lazy) improves both scalability of the service and availability of

An earlier version of the paper appeared in IEEE INFOCOM 2015.

$\mathrm{X}$. Li and D. Loguinov are with the Department of Computer Science and Engineering, Texas A\&M University, College Station, TX 77843 USA (e-mail: xiaoyong@cse.com; dmitri@cse.tamu.edu). D.B.H. Cline is with the Statistics Department at Texas A\&M University, College Station, TX 77843 USA (e-mail: dcline@stat.tamu.edu). the data, but at the expense of increased age of manipulated content [21], [43]. This model of operation has enjoyed ubiquitous deployment in the current Internet (e.g., HTTP, DNS, network monitoring, web caching, RSS feeds, stockticker aggregators, certain types of CDNs, sensor networks); however, it still poses many fundamental modeling challenges. Our goal is to study them in this paper.

\section{A. Motivation and Objectives}

Suppose a replica is a system whose goal is to synchronize against information sources, apply certain processing to downloaded content, and serve results to data consumers. One challenge of this architecture is that sources not just require pull-based operation, but also lack the ability to predict future updates, which makes real-time estimates of remaining object lifetime (i.e., TTL) unavailable to the replica.

As information evolves at the source, which we call data churn, the replica may become stale and provide responses to that do not reflect the true state of the system. In such cases, we assume that user satisfaction and system performance are directly rated to the amount of time by which the replica is lagging the source. To convert time units into cost, suppose the application applies some weight function $w(x)$ to the age of stale content to determine the penalty associated with a particular refresh policy and data-churn process. Then, the goal of the system is to optimize the expectation of penalty observed by a stream of arriving customers.

This problem has been considered in the context of webbased systems [4], [5], [8], [9], [10], [11], [15], [16], [17], [19], [22], [25], [26], [28], [29], [33], [35], [42]; however, all analytical results have predominantly assumed that the update process at each source was Poisson, with function $w(x)$ limited to either 1 or $x$. However, real systems driven by human behavior usually require more complex families of processes, often with heavy-tailed inter-update distributions, non-stationary dynamics, and slowly decaying correlation. Similarly, user sensitivity to outdated material may experience rapid increases for small $x$ and eventual saturation for larger $x$, in which case other weight functions might be more appropriate. Since application performance under general update processes and wider classes of $w(x)$ is currently open, we aim to fill this void below.

\section{B. Contributions}

Consider a single source driven by an update process $N_{U}$ and a single replica with the corresponding download process $N_{D}$, which is independent of $N_{U}$. Our first contribution is to propose a general framework for modeling staleness under arbitrary stochastic processes $\left(N_{U}, N_{D}\right)$. Since staleness age and various penalties derived from it are usually defined 


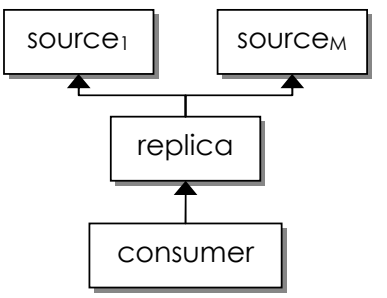

(a) aggregation

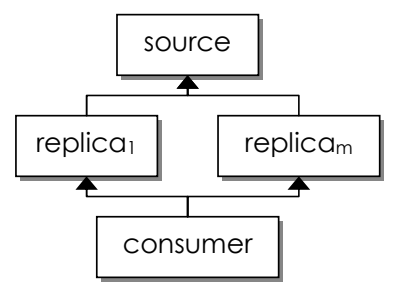

(b) backup/load-balancing
Fig. 1. System model (arrows signify the direction of requests for information).

in terms of sample-path averages [8], [9], [10], [11], [15], [16], [17], [25], [28], [29], [33], [35], [42], questions arise about their existence and possible variation across multiple realizations of the system. We address this issue by identifying the weakest set of conditions for which the distribution of staleness age exists and converges to the same deterministic value in every sample path.

Armed with these results, our second contribution is to model interaction between the age processes of $N_{U}$ and $N_{D}$. We show that for the results to be tractable, ages of the two processes examined at random times within a given sample path must be independent of each other. Interestingly, this condition does not automatically follow from independence of $N_{U}$ and $N_{D}$. Instead, we show that it translates into a form of ASTA (Arrivals See Time Averages) [27], where the download process $N_{D}$ must observe the sample-path distribution of update age.

Under the condition of age-independence, our third contribution is to derive the distribution of time by which the replica trails the source, the fraction of consumers that encounter a stale copy, the average number of missing updates from the replica at query time, and the general staleness cost under all suitable penalty functions $w(x)$. Our results involve simple closed-form expressions that are functions of limiting age distributions of both processes.

Our fourth contribution is to analyze conditions under which $N_{D}$ produces provably optimal penalty for a given download rate. We show that penalty reduces if and only if inter-refresh delays become stochastically larger in second order. This leads to constant synchronization delays being optimal under all $N_{U}$ and $w(x)$. This, however, presents problems in satisfying ASTA and creates a possibility of worst-case (i.e., 100\%) staleness due to phase-lock between the source and the replica. To this end, we discuss general requirements for ensuring that $N_{D}$ avoids these drawbacks while remaining optimal, or at least close to it.

We finish the paper with our last contribution that considers the practical aspects of staleness, including experimentation with Wikipedia page updates, error analysis of previous Poisson models, estimation of search-engine bandwidth requirements to maintain certain freshness, and generalization to multiple sources/replicas.

\section{STALENESS FORMULATION}

We start by explaining the underlying assumptions on the system, defining the various processes that determine informa-

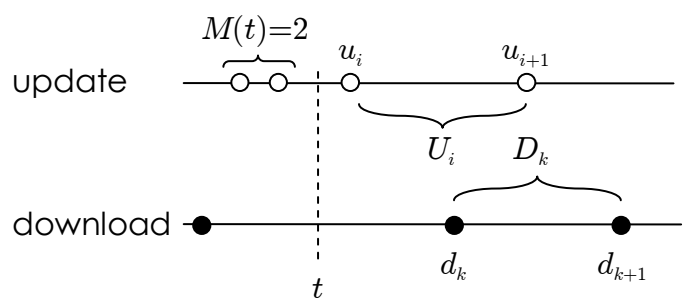

Fig. 2. Process notation.

tion flow, and specifying the metrics of interest.

\section{A. System Operation}

We assume a general model of distributed data generation, replication, and consumption shown in Fig. 1. During normal system operation, sources sustain random updates in response to external action (e.g., new posts in Facebook, traffic congestion in Google maps, Twitter feeds) or possibly some internal computation (e.g., MapReduce [13] indexing with periodic writes to disk). In either case, each update represents certain non-negligible information that manipulates the current state of the source.

Replicas operate independently of the sources and perform one of the two general functions shown in the figure - manyto-one aggregation in part (a) and one-to-many replication in (b). The former case arises when the replica executes certain processing on multiple objects to provide the consumer with results that cannot be obtained otherwise. These applications include search engines, data-centric computing, and various web front-ends that cache queries against back-end databases. The purpose of the latter case is to handle failover during source crashes and/or ensure scalable load distribution under heavy customer demand. Applications in this category include CDNs, large websites, data centers (e.g., Amazon EC2), and general distributed file systems.

The final element of Fig. 1 is the consumer, which sends a stream of requests that represent either queries for information or attempts to recover the most-recent state of the source after it has crashed.

\section{B. Updates and Synchronization}

We next model interaction between a single source and a single replica, which is a prerequisite to understanding system performance. Suppose the source undergoes updates at random times $0=u_{1}<u_{2}<\ldots$ and define $N_{U}(t)=\max \left\{i: u_{i} \leq\right.$ $t\}$ to be a stochastic process that counts the number of updates in $[0, t]$. When referring to the entire process, rather than its value at some point, we omit $t$ and write simply $N_{U}$.

For the replica, denote its random download instances by $0=d_{1}<d_{2}<\ldots$ and the corresponding point process by $N_{D}(t)=\max \left\{k: d_{k} \leq t\right\}$. This formulation neglects processing delays and treats all events as instantaneous. We additionally assume that both processes are simple (i.e., at most one point at any $t$ ) and independent of each other. Now, suppose the inter-update delays of $N_{U}$ are given by a random process $\left\{U_{i}\right\}_{i=1}^{\infty}$ and those of $N_{D}$ by $\left\{D_{k}\right\}_{k=1}^{\infty}$, which are 
illustrated in Fig. 2. Each of these sequences may be of fairly general nature, e.g., correlated and/or non-stationary.

\section{Cost of Staleness}

To understand the penalty of outdated content, suppose $M(t)$ counts the number of updates missing from the replica at time $t$ (e.g., in Fig. 2, $M(t)=2$ ). This is a discrete-state process that increments for each update and resets to zero for each synchronization.

Definition 1: A replica is called stale at time $t$ if $M(t)>0$. Otherwise, it is called fresh.

From the consumer's perspective, stale material reduces user satisfaction and lowers system performance, which needs to be translated into a cost metric that can be expressed via some known parameters of the system. The most basic penalty is the probability that the replica is stale at the time of request, i.e., $P(M(t)>0)$, which determines how often users see outdated information and/or fail to fully restore a crashed source The second obvious metric is the expected number of missing updates $E[M(t)]$, which measures the amount of lost information during a crash and estimates the difficulty in recreating it from the most recent checkpoint. This penalty is also important for Internet archiving applications that aim to capture every snapshot of the source [20] and situations when larger $M(t)$ may imply higher information divergence between the replica and the source.

More sophisticated cases are also possible. Suppose the source runs some computation, with updates representing certain intermediate states that are written to disk. A crash at time $t$ requires computation to be restarted, which means that the penalty is determined not by $M(t)$, but rather by the duration of the computation that was lost due to staleness. Services that charge per CPU time-unit (e.g., Amazon EC2) may want to optimize against this metric rather than $E[M(t)]$. Furthermore, if the difficulty of recovering each update from other storage is proportional to the delay since the update was made, then staleness cost may be based on the combined lag of all missing updates at time $t$.

Definition 2: For a stale replica at time $t$, define lags $L_{1}(t)>L_{2}(t)>\ldots>L_{M(t)}(t)$ to be backward delays to each unseen update.

This concept is illustrated in Fig. 3(a) for the first two lags. To keep the model general and cover the various options already seen in the literature [4], [5], [8], [9], we assume that the consumer is sensitive to either just lag $L_{1}(t)$, i.e., how long the source has been stale at time $t$, or the entire collection of lags $\left\{L_{1}(t), \ldots, L_{M(t)}(t)\right\}$, i.e., how long each uncaptured update has been stale. Since it is usually difficult to predict the value of information freshness to each customer, one requires a mapping from staleness lags to actual cost, which we assume is given by some non-negative weight function $w(x)$.

Definition 3: At time $t$, the source penalty is given by the weight of the delay since the replica was fresh last time:

$$
\eta(t)=\left\{\begin{array}{ll}
w\left(L_{1}(t)\right) & M(t)>0 \\
0 & \text { otherwise }
\end{array},\right.
$$

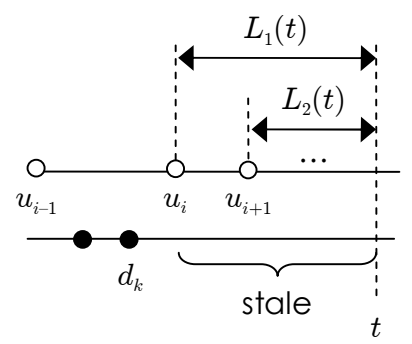

(a) staleness lags (b) age

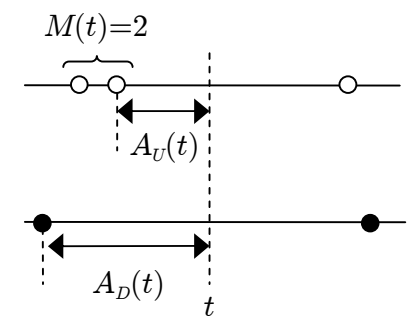

Fig. 3. Penalty lags and process age.

while the update penalty is given by the aggregate weight of all staleness lags:

$$
\rho(t)=\left\{\begin{array}{ll}
\sum_{i=1}^{M(t)} w\left(L_{i}(t)\right) & M(t)>0 \\
0 & \text { otherwise }
\end{array} .\right.
$$

For example, $w(x)=1$ produces the first two metrics discussed above, i.e., $P(M(t)>0)$ via $E[\eta(t)]$ and $E[M(t)]$ via $E[\rho(t)]$. Both (1) and (2) are random variables, which suggests that system performance should be assessed by their average values. But as neither $N_{U}$ nor $N_{D}$ is assumed to be stationary, the expected penalty requires additional elaboration. Instead of considering $E[\eta(t)]$ and $E[\rho(t)]$, which may depend on time $t$, it is more natural to replace them with sample-path averages [9]:

$$
\bar{\eta}=\lim _{T \rightarrow \infty} \frac{1}{T} \int_{0}^{T} \eta(t) d t \text { and } \bar{\rho}=\lim _{T \rightarrow \infty} \frac{1}{T} \int_{0}^{T} \rho(t) d t,
$$

where consumers are modeled as being equally likely to query the replica at any time in $[0, \infty)$.

\section{Relationship to Prior Work}

The majority of the literature on source penalty $\bar{\eta}$ is limited to Poisson $N_{U}$, either constant or exponential $D$, and $w(x)=$ 1 or $x$ [7], [8], [9], [11], [28], [36], [39], [42]. There has been only one attempt to model $\bar{\eta}$ under a general renewal process $N_{U}$, in which [39] assumed $w(x)=1$ and the entire sequence of refresh instances $\left\{d_{1}, d_{2}, \ldots\right\}$ was known. While appropriate in some cases, this model is difficult to evaluate in practice when $N_{D}$ is given by its statistical properties.

Update penalty $\bar{\rho}$ has received less exposure, with almost all papers considering Poisson updates and just constant $D$. This includes $w(x)=1$, where $\bar{\rho}$ is usually called divergence [19] or blur [14], with analysis available in [15], [16], [33], and $w(x)=x$, where $\bar{\rho}$ is known as additive age [24], aggregated age [25], delay [33], or simply cost [15]. Finally, $\bar{\rho}$ with a general $w(x)$ was called obsolescence cost in [16] and analyzed under a non-stationary Poisson $N_{U}$, but no closedform results were obtained.

The Poisson assumption on $N_{U}$ allows easy computation of the various metrics of interest. Outside these special cases, superposition of non-memoryless processes produces much more complex behavior. 


\section{Age Model}

While (3) is a convenient approach, there is a previously unnoticed obstacle with using it. Observe that (3) defines limits of sequences of random variables; however, it is unclear whether these limits exist, if they are finite, and under what conditions they are deterministic across all sample paths. We investigate these issues next.

\section{A. Main Framework}

We start by performing a convenient transformation of (3) to remove the integrals. Define $Q_{T}$ to be a uniform random variable in $[0, T]$, which models the random query time of consumers. Suppose $Q_{T}$ is independent of $N_{U}$ and $N_{D}$, in which case (3) is the limit of $E\left[\eta\left(Q_{T}\right) \mid N_{U}, N_{D}\right]$ and $E\left[\rho\left(Q_{T}\right) \mid N_{U}, N_{D}\right]$ as $T \rightarrow \infty$. We explicitly condition on processes $N_{U}, N_{D}$ to emphasize that all expectations and probabilities involving $Q_{T}$ are random variables (i.e., dependent on the pair of sample paths).

At each time $t$, suppose age processes $A_{U}(t)$ and $A_{D}(t)$, shown in Fig. 3(b), specify delays to the previous update and synchronization event, respectively. Using this notation and observing that $M(t)>0$ is equivalent to $A_{U}(t)<A_{D}(t)$, define an ON/OFF staleness process:

$$
S(t)=\left\{\begin{array}{ll}
1 & A_{U}(t)<A_{D}(t) \\
0 & \text { otherwise }
\end{array},\right.
$$

whose properties at random time $Q_{T}$ determine whether the consumer sees outdated information or not.

To analyze (4), our next topic is the behavior of $A_{U}\left(Q_{T}\right)$ and $A_{D}\left(Q_{T}\right)$ as $T \rightarrow \infty$, including existence of these limits and their relationship to $\left\{U_{i}\right\}_{i=1}^{\infty}$ and $\left\{D_{k}\right\}_{k=1}^{\infty}$.

\section{B. Assumptions}

We next aim to establish a minimal set of conditions under which analysis of staleness admits closed-form results. Consider a general point process $N$ with cycle lengths $\left\{X_{i}\right\}_{i=1}^{\infty}$, where each $X_{i} \sim F_{i}(x)$ is a random variable. In order for the age $A\left(Q_{T}\right)$ of this process to have a usable limiting distribution as $T \rightarrow \infty$, one must impose three constraints on $N$, which we discuss informally and motivate next, followed by a more rigorous, but functionally equivalent, definition.

The first restriction is that collection $\left\{X_{i}\right\}_{i=1}^{\infty}$ within each sample-path have some limiting distribution $F(x)$. The second prerequisite is that $F(x)$ be deterministic (i.e., equal in all sample-paths). Finally, the third condition is that an $o(1)$ fraction of cycles in $\left\{X_{i}\right\}_{i=1}^{n}$ not consume $\Omega(1)$ fraction of length as $n \rightarrow \infty$. This would be a problem because $F(x)$, being a limiting distribution, does not capture these intervals, but $Q_{T}$ still lands there with a non-diminishing probability.

Let $\mathbf{1}_{A}$ be an indicator variable of $A$ and $\bar{F}(x)=1-F(x)$ the complementary CDF (cumulative distribution function) of $F(x)$. We are now ready to summarize our discussion.

Definition 4: A process $N$ is called age-measurable if:

1) For all $x \geq 0$, except possibly points of discontinuity of the limit, sample-path distribution $H_{n}(x)$ of variables
$\left\{X_{1}, \ldots, X_{n}\right\}$ converges in probability as $n \rightarrow \infty$ :

$$
H_{n}(x):=\frac{1}{n} \sum_{i=1}^{n} \mathbf{1}_{X_{i} \leq x} \stackrel{P}{\rightarrow} F(x) ;
$$

2) Function $F(x)$ is deterministic with mean $0<\delta<\infty$;

3) The average cycle length converges to $\delta$ in probability as $n \rightarrow \infty$ :

$$
Z_{n}:=\frac{1}{n} \sum_{i=1}^{n} X_{i} \stackrel{P}{\rightarrow} \delta=\int_{0}^{\infty} \bar{F}(x) d x .
$$

Note that any renewal process $\left\{X_{i}\right\}_{i=1}^{\infty}$ satisfies this definition since all $F_{i}(x)$ are the same, which from the weak law of large numbers trivially leads to $F(x)=F_{i}(x)$ and $Z_{n} \rightarrow \delta$. Furthermore, condition (6) resembles mean-ergodicity, which is normally stated with a stronger type of convergence (e.g., mean-square or almost-sure) and only for stationary processes. For more general cases, the fact that indicator variables are uniformly bounded allows application of the Dominated Convergence Theorem (DCT) [31] to show that $F(x)$ is the limiting average of individual distributions:

$$
E\left[H_{n}(x)\right]=\frac{1}{n} \sum_{i=1}^{n} F_{i}(x) \rightarrow F(x) .
$$

If $\left\{X_{i}\right\}_{i=1}^{\infty}$ are uniformly bounded, then (6) follows from (5); however, this does not hold in general cases. In fact, many random variables used in practice (e.g., exponential and Pareto) are not bounded and thus require an explicit assumption that convergence in (6) take place. Additionally, even if this limit exists, it does not generally equal $\delta$, which is why we require that as well. Similarly, (5) does not follow from (6).

\section{Distribution}

To appreciate the different convergence results that follow, recall that $Z_{n} \rightarrow c$ in probability means that for all $\epsilon>0$ : $P\left(\left|Z_{n}-c\right|>\epsilon\right) \rightarrow 0$, i.e., the fraction of samples $Z_{n}$ that "stray away" from $c$ shrinks to zero, but the expected value of these outliers is potentially unlimited. Convergence in mean is defined as $E\left[\left|Z_{n}-c\right|\right] \rightarrow 0$, which shows that even outliers are well-bounded in expectation to some neighborhood of $c$. The latter convergence type is stronger, i.e., implies the former.

Theorem 1: For an age-measurable process $N$, the samplepath distribution of its age $A\left(Q_{T}\right)$ in points $Q_{T}$ uniformly distributed in $[0, T]$ converges in mean as $T \rightarrow \infty$ to the residual distribution of $F(x)$ :

$$
G(x):=\lim _{T \rightarrow \infty} P\left(A\left(Q_{T}\right)<x \mid N\right)=\frac{1}{\delta} \int_{0}^{x} \bar{F}(y) d y .
$$

Proof: Consider finite $T$ and some constant $b \geq 0$. Then, event $A\left(Q_{T}\right) \leq b$ is equivalent to the existence of some $k \geq 1$ such that $Q_{T}$ belongs to the $k$-th interval $\left[S_{k}, S_{k+1}\right)$, under the condition that starting point $S_{k} \leq T$ and age $Q_{T}-S_{k} \leq$ $b$. Defining $W_{k}=\min \left(\left(T-S_{k}\right)^{+}, X_{k}, b\right)$, where $(x)^{+}=$ $\max (x, 0)$, we get:

$$
P\left(A\left(Q_{T}\right)<b \mid N\right)=\sum_{k=1}^{\infty} P\left(S_{k} \leq Q_{T}<S_{k}+W_{k} \mid N\right) .
$$


Since $Q_{T}$ is uniform in $[0, T]$, the probability that it falls into an interval of length $W_{k}$ is simply $W_{k} / T$ :

$$
B_{T}:=P\left(A\left(Q_{T}\right)<b \mid N\right)=\sum_{k=1}^{N(T)} \frac{\min \left(T-S_{k}, X_{k}, b\right)}{T},
$$

where the upper limit is reduced from $\infty$ to $N(T)$ since $\left(T-S_{k}\right)^{+}=0$ for $k>N(T)$. Recalling that all probabilities and expectations are dependent on the sample path, it follows that $B_{T}$ is a random variable. Our goal below is to show it converges to a constant as $T \rightarrow \infty$. To this end, first observe that it can be bounded as:

$$
\frac{\sum_{k=1}^{N(T)-1} \min \left(X_{k}, b\right)}{\sum_{k=1}^{N(T)} X_{k}} \leq B_{T} \leq \frac{\sum_{k=1}^{N(T)} \min \left(X_{k}, b\right)}{\sum_{k=1}^{N(T)-1} X_{k}},
$$

where we use the fact that $T-S_{k} \geq X_{k}$ for all $k \leq N(T)-1$ and $T \in\left[\sum_{k=1}^{N(T)-1} X_{k}, \sum_{k=1}^{N(T)} X_{k}\right]$.

Next, notice that (5) implies that for all bounded, continuous functions $f(x)$ [31]:

$$
\frac{1}{n} \sum_{i=1}^{n} f\left(X_{i}\right) \stackrel{P}{\rightarrow} \int_{0}^{\infty} f(x) d F(x),
$$

which leads to:

$$
\lim _{T \rightarrow \infty} \frac{1}{N(T)} \sum_{k=1}^{N(T)-1} \min \left(X_{k}, b\right)=\int_{0}^{\infty} \min (y, b) d F(y) .
$$

Using (6), we also have:

$$
\lim _{T \rightarrow \infty} \frac{1}{N(T)} \sum_{k=1}^{N(T)-1} X_{k}=\lim _{n \rightarrow \infty} \frac{1}{n} \sum_{k=1}^{n-1} X_{k}=\delta .
$$

Since both bounds in (11) have the same limit, $B_{T}$ converges in probability to the ratio of (13) to (14):

$$
\frac{1}{\delta} \int_{0}^{\infty} \min (y, b) d F(y)=\frac{1}{\delta} \int_{0}^{b} \bar{F}(y) d y,
$$

where the second integral follows from expanding the min function and integrating by parts. Observing that $B_{T} \leq 1$ is bounded, we can invoke the Dominated Convergence Theorem [31] to conclude that $E\left[B_{T}\right]$ converges to (15). Finally, as $B_{T}$ converges in probability to the same result as $E\left[B_{T}\right]$, convergence in mean immediately follows [31].

Interestingly, (5)-(6) are not only sufficient as demonstrated by this theorem, but also necessary for $G(x)$ to exist and equal the right side of (8). Necessity is proven by well-known counter-examples in probability theory [38].

\section{Expectation}

While Theorem 1 establishes when $A\left(Q_{T}\right)$ has a limiting distribution, convergence of expectation $E\left[A\left(Q_{T}\right) \mid N\right]$ or suitability of $G(x)$ for computing it are not guaranteed. Furthermore, given that consumers may apply generic weights $w(x)$ to the various age-related metrics, it makes sense to ask when $E\left[w\left(A\left(Q_{T}\right)\right) \mid N\right]$ exists as $T \rightarrow \infty$.

To build intuition for the next result, assume $X \sim F(x)$ is a non-negative variable and define its age $A$ to be a random variable with $\operatorname{CDF} G(x)$ in (8). Then, we are interested in the relationship between $E[w(A)]$ and $X$. To this end, suppose for any locally integrable function $w(x)$, we set $w_{1}(x)=w(x)$ and then recursively integrate the result $n-1$ times to define:

$$
w_{n}(x):=\int_{0}^{x} w_{n-1}(y) d y .
$$

Using integration by parts in Lebesgue-Stieltjes integrals and keeping in mind that $w_{n+1}(0)=0$ for $n \geq 1$ :

$$
E\left[w_{n+1}(X)\right]=\int_{0}^{\infty} w_{n}(x) \bar{F}(x) d x=E\left[w_{n}(A)\right] E[X] .
$$

Therefore, in order for $E[w(A)]$ to exist, one must ensure that both $E\left[w_{2}(X)\right]$ and $E[X]$ do. Note that the latter does so by (6), but the former requires an additional constraint.

Definition 5: A point process $N$ is called age-measurable by weight function $w(x)$ if it is age-measurable and

$$
\frac{1}{n} \sum_{i=1}^{n} w_{2}\left(X_{i}\right) \stackrel{P}{\rightarrow} \int_{0}^{\infty} w_{2}(x) d F(x)<\infty .
$$

Note that age-measurable by a constant is equivalent to simply age-measurable since in that case (18) becomes (6). We omit the proof of the next result as it follows that of Theorem 1 pretty closely. The only difference is that since $w(x)$ may be unbounded, convergence is stated in probability rather than in mean.

Theorem 2: For a process $N$ that is age-measurable by $w(x)$, the sample-path expectation of $w\left(A\left(Q_{T}\right)\right)$ converges in probability as $T \rightarrow \infty$ :

$$
\lim _{T \rightarrow \infty} E\left[w\left(A\left(Q_{T}\right)\right) \mid N\right]=\int_{0}^{\infty} w(x) d G(x) .
$$

From this point on, we omit explicit conditioning on the sample-path since results do not depend on $N$ for agemeasurable processes. However, we keep in mind that all probabilities and expectations involving $Q_{T}$ are still taken in the sample-path sense.

\section{STALeness Cost}

This section models the probability of staleness and expected cost under both penalty metrics defined earlier.

\section{A. Age Independence}

We now return to examining (4). In order to determine when the replica is stale, one requires comparison of $A_{U}\left(Q_{T}\right)$ with $A_{D}\left(Q_{T}\right)$, which may not be independent random variables, even if $N_{U}$ and $N_{D}$ are. For example, suppose $\left\{U_{i}\right\}_{i=1}^{\infty}$ and $\left\{D_{k}\right\}_{i=1}^{\infty}$ are iid variables that equal 1 or 2 with probability $1 / 2$ each. As $T \rightarrow \infty$, the distribution of $A_{D}\left(Q_{T}\right)$ becomes a mixtures of two uniform variables in $[0,1]$ and $[0,2]$. However, conditioning on $A_{U}\left(Q_{T}\right)=y$ shifts the mass of refresh age $A_{D}\left(Q_{T}\right)$ to just three discrete points $y-1, y, y+1$, clearly showing that the two ages are dependent.

To prevent such cases, which is called phase-lock [3], the safest solution is to require that $N_{D}$ implement a download policy that ensures independence of the two ages. In that case, conditions such as ASTA (Arrivals See Time Averages) [27] 
must apply to the age of one process when sampled by the arrival points of the other. This issue is delayed until a later section, but for the time being we define more clearly what independence of $A_{U}\left(Q_{T}\right)$ and $A_{D}\left(Q_{T}\right)$ means.

Specifically, suppose $N_{U}$ and $N_{D}$ are age-measurable. Then, let $F_{U}(x)$ and $F_{D}(x)$ be respectively the limiting $\mathrm{CDF}$ functions of interval lengths defined in (5), with the corresponding average rates $\mu$ and $\lambda$, i.e.,

$$
\frac{1}{\mu}=\int_{0}^{\infty} \bar{F}_{U}(x) d x \text { and } \frac{1}{\lambda}=\int_{0}^{\infty} \bar{F}_{D}(x) d x .
$$

Further, let $U \sim F_{U}(x)$ and $D \sim F_{D}(x)$ be random update and download cycle lengths. Similarly, suppose $G_{U}(x)$ and $G_{D}(x)$ are the limiting CDFs of age from (8), with lower-case functions $g_{U}(x)$ and $g_{D}(x)$ representing the corresponding PDFs. When the necessary limits exist, let $A_{U} \sim G_{U}(x)$ and $A_{D} \sim G_{D}(x)$ represent the two random ages as $T \rightarrow \infty$.

Definition 6: Two point processes $N_{U}$ and $N_{D}$ are called age-independent if they are age-measurable and $\forall x, y \geq 0$ :

$$
\lim _{T \rightarrow \infty} P\left(A_{D}\left(Q_{T}\right)<x \mid A_{U}\left(Q_{T}\right)=y\right)=G_{D}(x) .
$$

If either $N_{U}$ or $N_{D}$ is Poisson, (21) is guaranteed from PASTA (Poisson Arrivals See Time Averages) [40], which explains why prior work did not encounter these nuances. In more general cases, (21) can be expressed in a more convenient form as shown next.

Theorem 3: Formulation (21) implies that $A_{D}(t)$ sampled in update points of $N_{U}$ produces a sequence of random variables that converges in distribution to $G_{D}(x)$ :

$$
\lim _{T \rightarrow \infty} \frac{1}{N_{U}(T)} \sum_{i=1}^{N_{U}(T)} \mathbf{1}_{A_{D}\left(u_{i}\right)<x}=G_{D}(x) .
$$

Proof: First define $d(y, T)$ to be the number of points in $[0, T]$ where $A_{U}(t)=y$ occurs. Recalling that $u_{1}=0$, this can be expressed as:

$$
d(y, T)=\sum_{i=1}^{N_{U}(T)} \mathbf{1}_{U_{i} \geq y, u_{i}+y \leq T}=\sum_{i=1}^{N_{U}(T)} \mathbf{1}_{U_{i} \geq y} \cdot \mathbf{1}_{u_{i}+y \leq T} .
$$

Next, let $c(y, T)$ be the number of these points in which the download age $A_{D}$ is smaller than $x$ :

$$
\begin{aligned}
c(y, T) & =\sum_{i=1}^{N_{U}(T)} \mathbf{1}_{U_{i} \geq y, u_{i}+y \leq T, A_{D}\left(u_{i}+y\right)<x} \\
& =\sum_{i=1}^{N_{U}(T)} \mathbf{1}_{U_{i} \geq y} \cdot \mathbf{1}_{u_{i}+y \leq T} \cdot \mathbf{1}_{A_{D}\left(u_{i}+y\right)<x} .
\end{aligned}
$$

Noticing that

$$
P\left(A_{D}\left(Q_{T}\right)<x \mid A_{U}\left(Q_{T}\right)=y\right)=\frac{c(y, T)}{d(y, T)},
$$

we get using (21):

$$
G_{D}(x)=\lim _{T \rightarrow \infty} \lim _{y \rightarrow 0} \frac{c(y, T)}{d(y, T)}=\lim _{T \rightarrow \infty} \frac{1}{N_{U}(T)} \sum_{i=1}^{N_{U}(T)} \mathbf{1}_{A_{D}\left(u_{i}\right)<x},
$$

which is the same as (22).

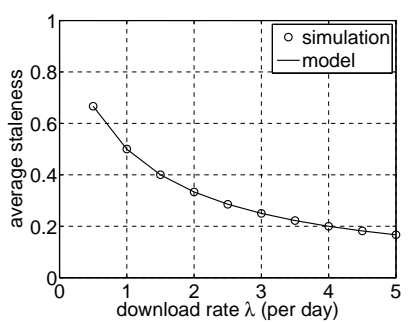

(a) Pareto $U$, constant $D$

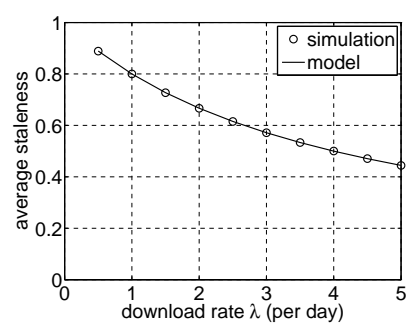

(b) constant $U$, Pareto $D$
Fig. 4. Examination of (25) under $\mu=2$.

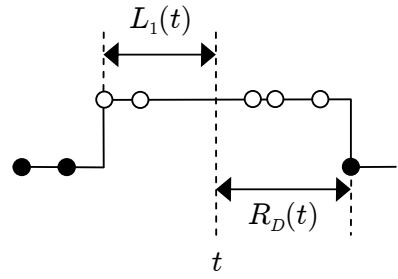

(a) staleness process $S(t)$

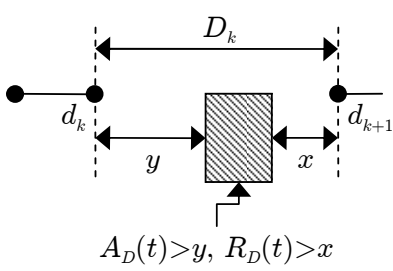

(b) reward of each cycle
Fig. 5. Visualizing the proof of Theorem 5.

\section{B. Preliminaries}

Our first objective is to derive the probability of staleness.

Theorem 4: Assuming that $N_{U}$ and $N_{D}$ are age-independent, the probability of staleness at time $Q_{T}$ converges in mean as $T \rightarrow \infty$ to:

$$
P\left(S\left(Q_{T}\right)=1\right) \rightarrow p:=\mu \int_{0}^{\infty} \bar{F}_{U}(y) \bar{G}_{D}(y) d y .
$$

Proof: Due to the existence and independence of $A_{U}\left(Q_{T}\right)$ and $A_{D}\left(Q_{T}\right)$ in the limit, we immediately obtain:

$$
p=P\left(A_{D}>A_{U}\right)=\int_{0}^{\infty} \bar{G}_{D}(y) d G_{U}(y) .
$$

Expanding $d G_{U}(y)=\mu \bar{F}_{U}(y) d y$ leads to the result.

To perform a self-check against prior results with Poisson $N_{U}$, observe that (25) simplifies to $p=1-\lambda\left(1-e^{-\mu / \lambda}\right) / \mu$ under constant $D$ and $\mu /(\mu+\lambda)$ under exponential $D$, which are consistent with [9], [11]. Simulations in Fig. 4 examine model accuracy in more interesting cases of general renewal processes. We use Pareto CDF $1-(1+x / \beta)^{-\alpha}$ with $\alpha=3$ and mean $\beta /(\alpha-1)=\beta / 2$. Observe in the figure that the model matches simulations very well, with constant download intervals performing significantly better against Pareto update cycles in (a) than the other way around in (b). For example, synchronizing pages at their update rate (i.e., $\lambda=\mu=2$ ) serves stale copies with probability $33 \%$ in the former case and $66 \%$ in the latter. Furthermore, for the same $p$, the scenario in (a) requires roughly 4 times less bandwidth than in (b).

The next intermediate result is the distribution of the first lag $L_{1}\left(Q_{T}\right)$, which relies on $p$ in (25).

Theorem 5: If $N_{U}$ and $N_{D}$ are age-independent, the CDF of $L_{1}\left(Q_{T}\right)$ converges in mean as $T \rightarrow \infty$ to:

$$
F_{L}(x)=1-\frac{\mu}{p} \int_{0}^{\infty} \bar{F}_{U}(y) \bar{G}_{D}(x+y) d y .
$$




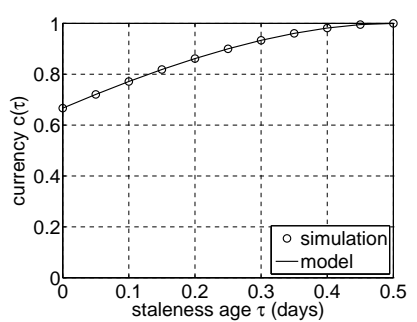

(a) Pareto $U$, constant $D$

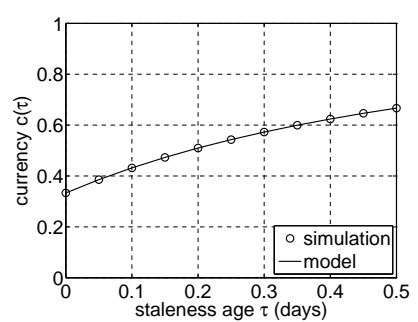

(b) constant $U$, Pareto $D$

Fig. 6. Examination of (30) under $\lambda=\mu=2$.

Proof: Consider the ON/OFF staleness process in Fig. 5 (a) and suppose the query time $t$ falls in the ON period. Then, since $t$ is uniformly random within this cycle, the backward delay $L_{1}(t)$ is symmetrical to the forward (residual) delay $R_{D}(t)$, meaning they have the same distribution. Note that it is important to condition on $A_{D}(t)>A_{U}(t)$ since residual $R_{D}(t)$ depends on age $A_{D}(t)$, i.e.,

$$
P\left(L_{1}(t)>x\right)=P\left(R_{D}(t)>x \mid A_{D}(t)>A_{U}(t)\right) .
$$

Since the ages of $N_{U}$ and $N_{D}$ are independent, we can condition on $A_{U}(t)=y$ for any $t$ without impacting the distribution of $A_{D}(t)$ or $R_{D}(t)$. Following the proof of Theorem 1 , define $L_{k}=d_{k}+y$ and $M_{k}=\min \left(T, d_{k+1}-x\right)$ to be the lower/upper boundaries within synchronization interval $k$ such that if $t \in\left[L_{k}, M_{k}\right]$ then $R_{D}(t)>x$ and $A_{D}(t)>y$. See Fig. 5(b) for an illustration.

Define $C_{T}=P\left(L_{1}\left(Q_{T}\right)>x \mid A_{U}\left(Q_{T}\right)=y\right)$ and observe that it converges as $T \rightarrow \infty$ :

$$
\begin{aligned}
C_{T} & =\frac{1}{p T} \sum_{k=1}^{\infty} P\left(L_{k} \leq Q_{T} \leq M_{k}\right)=\frac{1}{p T} \sum_{k=1}^{N(T)}\left(D_{k}-(x+y)\right)^{+} \\
& \rightarrow \frac{\lambda}{p} \int_{0}^{\infty} \max (z-(x+y), 0) d F_{D}(z) \\
& =-\frac{\lambda}{p} \int_{x+y}^{\infty}(z-(x+y)) d \bar{F}_{D}(z)=\frac{\lambda}{p} \int_{x+y}^{\infty} \bar{F}_{D}(z) d z \\
& =\frac{1}{p} \int_{x+y}^{\infty} g_{D}(z) d z=\frac{\bar{G}_{D}(x+y)}{p} .
\end{aligned}
$$

Unconditioning $A_{U}\left(Q_{T}\right)$ and keeping in mind that its distribution is well-defined as $T \rightarrow \infty$, we get (27).

Theorem 5 allows a simple expression for the fraction of requests $c(\tau)$ that observe content outdated by less than $\tau$ time units, which was called $\beta$-currency in [4] and $\Delta$-consistency in [36]. This can be expressed as:

$$
\left.c(\tau)=1-\bar{F}_{L}(\tau) p=\int_{0}^{\infty} g_{U}(y) G_{D}(\tau+y)\right) d y
$$

which conveniently simplifies to $P\left(A_{D}-A_{U}<\tau\right)$, where $A_{D}-A_{U}$ is the generalized lag between the replica and the source, i.e., non-positive values mean fresh states. Fig. 6 compares (30) to simulations using $\lambda=\mu$. As seen in the figure, this page retrieved at a random time is stale by less than $\tau=0.4$ days (9.6 hours) with probability $c(\tau)=98 \%$ in the first case and $62 \%$ in the second.

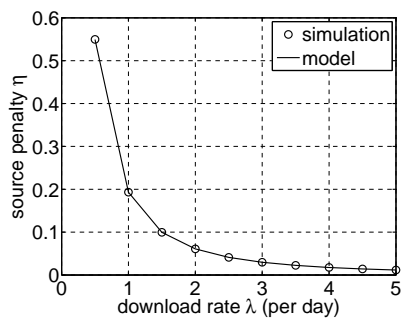

(a) Pareto $U$, constant $D$

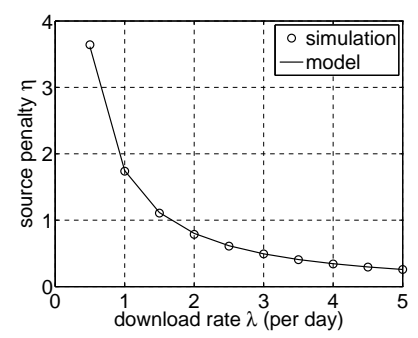

(b) constant $U$, Pareto $D$
Fig. 7. Examination of (31) under $w(x)=x, \mu=2$.

\section{Source Penalty}

We are now ready to derive a general formula for $\bar{\eta}$.

Theorem 6: If $N_{U}$ and $N_{D}$ are age-independent, while $N_{D}$ is age-measurable by $w(x)$, the source penalty converges in probability to:

$$
\bar{\eta}=\lambda \mu \int_{0}^{\infty} \bar{F}_{U}(y) \int_{0}^{\infty} w(x) \bar{F}_{D}(x+y) d x d y .
$$

Proof: First, observe that

$$
\bar{\eta}=E\left[w\left(L_{1}\right)\right] P\left(A_{D}>A_{U}\right),
$$

where $L_{1} \sim F_{L}(x)$. Working back from (27), the tail CDF of $L_{1}$ can be written more compactly as:

$$
P\left(L_{1}>x\right)=P\left(A_{D}-A_{U}>x \mid A_{D}>A_{U}\right) .
$$

Since $L_{1}=A_{D}-A_{U}>0$, conditioned on $A_{D}>A_{U}$, it suffices that only process $N_{D}$ be measurable by $w(x)$. In that case:

$$
\bar{\eta}=E\left[w\left(L_{1}\right)\right] P\left(A_{D}>A_{U}\right)=p \int_{0}^{\infty} w(x) d F_{L}(x),
$$

or equivalently:

$$
\bar{\eta}=\int_{0}^{\infty} g_{U}(y) \int_{0}^{\infty} w(x) g_{D}(x+y) d x d y .
$$

which immediately leads to (31) after expansion of $g_{U}(x)=$ $\mu \bar{F}_{U}(x)$ and $g_{D}(x+y)=\lambda \bar{F}_{D}(x+y)$.

With $w(x)=1$, (31) reduces to staleness probability $p$ already discussed above. For the other case $w(x)=x$ seen in the literature, we obtain the expected staleness age $\bar{\eta}=E\left[L_{1}(t)\right] p$ by which the replica trails the source. Under Poisson $N_{U}$ and constant $D$, we get from (31):

$$
\bar{\eta}=\frac{1}{2 \lambda}-\frac{1}{\mu}+\frac{\lambda\left(1-e^{-\mu / \lambda}\right)}{\mu^{2}},
$$

and when both distributions are exponential:

$$
\bar{\eta}=\frac{\mu}{\lambda(\lambda+\mu)} .
$$

These special cases are consistent with [8]. Simulations in Fig. 7 additionally confirm that (31) is accurate under general renewal processes. Also observe in the figure that the combination in (b) continues to offer inferior performance to that in (a); however, the difference between the two scenarios is now more pronounced. For example, using the same $\lambda=\mu$ considered earlier, search-engine clients encounter indexing 
results outdated on average by 0.06 days ( 1.5 hours) in the left subfigure and by 0.8 days (19 hours) in the right. This example shows how drastically the cost changes based on the shape of $F_{U}(x)$ and $F_{D}(x)$, which emphasizes the importance of utilizing models that can accurately handle any underlying processes $\left(N_{U}, N_{D}\right)$.

We now offer a more intuitive look at source penalty. Modifying $w(x)$ to be zero for negative $x$, we can rewrite (31) in a more compact form:

$$
\bar{\eta}=E\left[w\left(A_{D}-A_{U}\right)\right]=\lambda E\left[w_{2}\left(D-A_{U}\right)\right] .
$$

This result shows that $\bar{\eta}$ is determined by the positive deviation of the generalized lag $A_{D}-A_{U}$ from zero, or equivalently by that of $D-A_{U}$, where the weight applied to each deviation is given respectively by $w(x)$ and $w_{2}(x)$. The only caveat is that simplification (38) requires weight functions that can explicitly handle negative arguments, e.g., a constant penalty would be $w(x)=\mathbf{1}_{x \geq 0}$ rather than just $w(x)=1$. Throughout the rest of the paper, we avoid the extra notation dealing with $x<0$, but keep this in mind.

\section{Update Penalty}

Unlike the previous section, we next show that $\bar{\rho}$ admits a much simpler result that depends only on the mean update rate $\mu$ rather than the entire distribution $F_{U}(x)$. This was first observed through simulations in [15] for constant interval lengths $D$, but no explanation or extension to other $F_{D}(x)$ was offered.

Theorem 7: Assuming $N_{U}$ and $N_{D}$ are age-independent, while $N_{D}$ is age-measurable by $w_{2}(x)$, the update penalty converges in probability to:

$$
\bar{\rho}=\mu E\left[w_{2}\left(A_{D}\right)\right]=\lambda \mu E\left[w_{3}(D)\right] .
$$

Proof: Using Lebesgue-Stieltjes integrals and treating point processes as measures, we can re-write (2) as:

$$
\rho(t)=\int_{t-A_{D}(t)}^{t} w(t-s) d N_{U}(s)
$$

Taking the expectation along each sample path:

$$
\begin{aligned}
\bar{\rho} & =\lim _{T \rightarrow \infty} E\left[\int_{Q_{T}-A_{D}\left(Q_{T}\right)}^{Q_{T}} w\left(Q_{T}-s\right) d N_{U}(s)\right] \\
& =\lim _{T \rightarrow \infty} \frac{1}{T} \int_{0}^{T} \int_{t-A_{D}(t)}^{t} w(t-s) d N_{U}(s) d t \\
& =\lim _{T \rightarrow \infty} \frac{1}{T} \int_{0}^{T} w_{2}\left(A_{D}(t)\right) d N_{U}(t) \\
& =\lim _{T \rightarrow \infty} \frac{1}{T} \sum_{i=1}^{N_{U}(T)} w_{2}\left(A_{D}\left(u_{i}\right)\right) .
\end{aligned}
$$

Applying (22), the sequence $\left\{A_{D}\left(u_{1}\right), A_{D}\left(u_{2}\right), \ldots\right\}$ sampled in update points $\left\{u_{i}\right\}$ converges in distribution to that of $A_{D}\left(Q_{T}\right)$ as $T \rightarrow \infty$. Then, (41) becomes:

$$
\bar{\rho}=\lim _{T \rightarrow \infty} \mu E\left[w_{2}\left(A_{D}\left(Q_{T}\right)\right)\right] .
$$

Since $N_{D}$ is $w_{2}(x)$-measurable, (19) shows that this expectation converges and its limit equals $\mu E\left[w_{2}\left(A_{D}\right)\right]$. By (17), this is also $\lambda \mu E\left[w_{3}(D)\right]$.

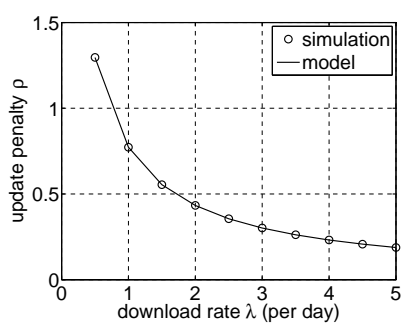

(a) Pareto $U$, constant $D$

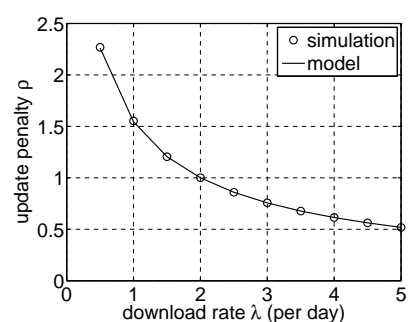

(b) constant $U$, Pareto $D$
Fig. 8. Examination of (45) and (46) under $\mu=2$.

To perform a sanity check, consider Poisson $N_{U}$ and constant $D$. Then, (39) produces $\bar{\rho}=\mu /(2 \lambda)$ for $w(x)=1$ and $\mu /\left(6 \lambda^{2}\right)$ for $w(x)=x$, both of which match previously known results [24], [33], [42]. Generalizing these two cases to exponential $D$, we obtain from (39) respectively $\mu / \lambda$ and $\mu / \lambda^{2}$. Interestingly, this shows that switching downloads from constant intervals to exponential doubles the number of missing updates and sextuples their combined age.

For $w(x)=1$, a simple closed-form expression is possible for all $D$ :

$$
E[M(t)]=\frac{\lambda \mu E\left[D^{2}\right]}{2}=\frac{\mu}{2 \lambda}\left(1+\lambda^{2} \operatorname{Var}[D]\right) .
$$

For example, Pareto $D$ produces in (43):

$$
E[M(t)]=\frac{\mu(\alpha-1)}{\lambda(\alpha-2)},
$$

which for $\alpha=3$ is quadruple that of constant $D$ and double that of exponential $D$. Another peculiar case is $\alpha \rightarrow 2$, where $E[M(t)]$ tends to infinity regardless of $N_{U}$. In fact, the update process itself may exhibit $\operatorname{Var}[U]=\infty$, but the expected number of updates by which the replica falls behind will still become unbounded as $\alpha$ approaches 2 .

Since source penalty $\bar{\rho}$ sums up the ages of all missing updates, it allows usage of decaying functions $w(x)$ such that their integral is increasing. We demonstrate this effect using $w(x)=1 /(1+x)$, for which $w_{2}(x)=\log (1+x)$. This cost function increases rapidly for small $x$, but then becomes less sensitive to staleness as the age of replicated content grows. Since $w_{3}(x)=(1+x) \log (1+x)-x$, constant $D$ yields:

$$
\bar{\rho}=\mu[(\lambda+1) \log (1+1 / \lambda)-1] .
$$

For $D \sim \operatorname{Pareto}(\alpha, \beta)$ with $\alpha=3$ and $\beta=2 / \lambda$, we get:

$$
\bar{\rho}=2 \mu\left\{\begin{array}{ll}
\frac{2 \log (2 / \lambda)-2+\lambda}{(\lambda-2)^{2}} & \lambda \neq 2 \\
0.25 & \lambda=2
\end{array} .\right.
$$

Fig. 8 confirms that both models are accurate, with constant $D$ enjoying a $60 \%$ lower penalty compared to Pareto.

\section{Optimality}

Motivated by (43) and consistently worse performance of Pareto $D$, the goal of this section is to understand the impact, if any, of $\operatorname{Var}[D]$ on penalty and determine whether there exists an optimal distribution $F_{D}(x)$ that, for a fixed download budget $\lambda$, provably results in the lowest cost for all $N_{U}$ and all suitable functions $w(x)$. 


\section{A. Stochastic Dominance}

We start with general concepts from economics and game theory that are useful for understanding optimality. For two non-negative random variables $X \sim F_{X}(x)$ and $Y \sim F_{Y}(x)$, let their CDF difference be:

$$
H(x)=F_{Y}(x)-F_{X}(x),
$$

whose generalization $H_{n}(x)$ is given by (16). Then, we have the following definition.

Definition 7: Variable $X$ is said to stochastically dominate $Y$ in $n$-th order, which we write as $X \geq_{s t}^{n} Y$, if $H_{n}(x) \geq 0$ for all $x \in \mathbb{R}$.

This concept is important because desirable characteristics of $D$ can be inferred from those of $A_{D}$, as shown next.

Lemma 1: Assume $E[X]=E[Y]$ and $n \geq 2$. Then, $X$ stochastically dominates $Y$ in $n$-th order, i.e., $X \geq_{s t}^{n} Y$, iff the age of $Y$ stochastically dominates the age of $X$ in $(n-1)$ st order, i.e., $A_{Y} \geq_{s t}^{n-1} A_{X}$.

Proof: Let $G_{X}(x)$ and $G_{Y}(x)$ be the CDF of $A_{X}$ and $A_{Y}$, respectively. Define:

$$
J(x)=G_{Y}(x)-G_{X}(x),
$$

which can be expressed using $H_{2}(x)$ as:

$$
\begin{aligned}
J(x) & =\frac{\int_{0}^{x}\left(1-F_{Y}(x)\right) d y}{E[Y]}-\frac{\int_{0}^{x}\left(1-F_{X}(y)\right) d y}{E[X]} \\
& =\frac{\int_{0}^{x}\left(F_{X}(y)-F_{Y}(y)\right) d y}{E[X]}=-\frac{1}{E[X]} H_{2}(x) .
\end{aligned}
$$

Integrating both sides $n-2$ additional times leads to:

$$
J_{n-1}(x)=-\frac{1}{E[X]} H_{n}(x) .
$$

From this and Definition 7, it follows that $X \geq_{s t}^{n} Y$ implies $A_{Y} \geq_{s t}^{n-1} A_{X}$ and vice versa.

As given by the next lemma, first-order stochastic dominance allows one to determine the relationship between expected utilities $E[w(X)]$ and $E[w(Y)]$. While it is possible to establish a more general version of this result using $n$-th order dominance, it would restrict $w(x)$ to a narrower class of functions and thus would be less useful in practice.

Lemma 2: Condition $X \geq \geq_{s t}^{1} Y$ holds iff for all non-decreasing functions $w(x)$ it follows that $E[w(X)] \geq E[w(Y)]$.

\section{B. Penalty Analysis}

Returning to the topic of information staleness, our goal is to determine the condition under which both types of penalty can be reduced without changing the refresh rate $\lambda$. Define $\bar{\eta}\left(D_{1}\right)$ and $\bar{\eta}\left(D_{2}\right)$ to be the source penalties corresponding to random synchronization intervals $D_{1}$ and $D_{2}$. For the opposite problem, i.e., finding the worst update distribution for a given $\mu$, define $\bar{\eta}\left(U_{1}\right)$ and $\bar{\eta}\left(U_{2}\right)$ to be the penalties that correspond to update intervals $U_{1}$ and $U_{2}$.

The next result shows that stochastic (rather than variance) ordering is needed to improve staleness penalty. Define $w(x)$ to be a measure if it is non-negative, non-decreasing, and rightcontinuous with $w(x)=0$ for $x<0$.
Theorem 8: For a given $N_{U}$ and fixed download rate $\lambda$, $D_{1} \geq_{s t}^{2} D_{2}$ iff $\bar{\eta}\left(D_{1}\right) \leq \bar{\eta}\left(D_{2}\right)$ for all measures $w(x)$. Similarly, with a given $N_{D}$ and fixed $\mu, U_{1} \geq_{s t}^{2} U_{2}$ iff $\bar{\eta}\left(U_{1}\right) \geq \bar{\eta}\left(U_{2}\right)$ for all measures $w(x)$.

Proof: Using (38), observe that $\bar{\eta}=E\left[w\left(A_{D}-A_{U}\right)\right]$ is fully determined by the properties of variable $X=A_{D}-A_{U}$. For a fixed $A_{U}$, it is not difficult to show that $X$ becomes stochastically smaller in first order iff $A_{D}$ does. Applying Lemmas $1-2$, this means that penalty $\bar{\eta}$ gets smaller iff $D$ increases stochastically in second order.

Similarly, for a fixed $A_{D}, X$ gets stochastically larger in first order iff $A_{U}$ becomes stochastically smaller. Again applying Lemmas $1-2$, penalty $\bar{\eta}$ increases iff $U$ becomes stochastically larger in second order.

A similar result holds under update penalty $\bar{\rho}$. Note that $F_{U}(x)$ has no impact here and the result holds for all $w(x)$.

Theorem 9: For a given $N_{U}$ and fixed $\lambda, D_{1} \geq_{s t}^{2} D_{2}$ iff $\bar{\rho}\left(D_{1}\right) \leq \bar{\rho}\left(D_{2}\right)$ for all $w(x)$.

Proof: Since $\bar{\rho}=\mu E\left[w_{2}\left(A_{D}\right)\right]$, where $w_{2}(x)$ is a measure for all non-negative $w(x)$, Lemmas 1-2 yield that $\bar{\rho}$ decreases iff $D$ gets stochastically larger in second order.

The preceding results set up motivation to ask the question of whether there exists a distribution that dominates all others in second order. We answer this next.

Theorem 10: For a given mean, a constant stochastically dominates all other random variables in second order.

Proof: Suppose $l$ is the fixed mean of all distributions under consideration. Let $F_{X}(x)=\mathbf{1}_{x>l}$ be the CDF of a constant and $F_{Y}(x)$ be the CDF of another random variable $Y$ such that $E[Y]=l$. Our goal is to show that $H_{2}(x) \geq 0$.

When $x \leq l$, we have trivially:

$$
H_{2}(x)=\int_{0}^{x}\left(F_{Y}(y)-F_{X}(y)\right) d y=\int_{0}^{x} F_{Y}(y) d y \geq 0 \text {. }
$$

For $x>l$, we get:

$$
\begin{aligned}
H_{2}(x) & =\int_{0}^{l} F_{Y}(y) d y+\int_{l}^{x}\left(F_{Y}(y)-1\right) d y \\
& =l+\int_{0}^{x} F_{Y}(y) d y-x=l-\int_{0}^{x}\left(1-F_{Y}(y)\right) d y \\
& \geq l-\int_{0}^{\infty}\left(1-F_{Y}(y)\right) d y=0
\end{aligned}
$$

where we use the fact that $l=\int_{0}^{\infty}\left(1-F_{Y}(y)\right) d y$.

This leads to the main result of this section.

Corollary 1: Constant inter-synchronization delays are optimal under both $\bar{\eta}$ and $\bar{\rho}$, all suitable weights $w(x)$, and all update processes $N_{U}$.

This allow us to resolve the relationship between the variance of $D$ and penalty. If $E\left[D_{1}\right]=E\left[D_{2}\right]$, then $D_{1} \geq_{s t}^{2} D_{2}$ implies $\operatorname{Var}\left[D_{1}\right] \leq \operatorname{Var}\left[D_{2}\right]$, but the opposite is not true. This shows that for a given download rate, just reducing the variance of refresh intervals, without enforcing $D_{1} \geq_{s t}^{2} D_{2}$, is insufficient to improve the penalty across all functions $w(x)$. As an example, recall the special case of $\bar{\rho}$ with $w(x)=1$ in (43), where the penalty was reduced iff the variance of $D$ was; however, no such causality exists for $w(x)=x$ or $\log (1+x)$. On the other hand, if reduction in penalty holds for 
all measures $w(x)$, then stochastic ordering between $D_{1}$ and $D_{2}$ follows and thus variance has to decrease (i.e., ordering of variances is necessary, but not sufficient).

\section{Phase-Lock}

Even though constant $D$ is optimal from the staleness perspective, it unfortunately fails to guarantee age-independence (21) against all underlying $N_{U}$. We now deal with principles related to ASTA (Arrivals See Time Averages) [27], placing them in our context. In general, ASTA can be viewed as a condition that allows discrete and continuous sample-path averages of a stochastic process $X(t)$ to be equal almost surely:

$$
\lim _{n \rightarrow \infty} \frac{1}{n} \sum_{k=1}^{n} X\left(t_{k}\right)=\lim _{T \rightarrow \infty} \frac{1}{T} \int_{0}^{T} X(t) d t .
$$

Let $X(t)=\mathbf{1}_{A_{D}(t)<x}$ and $t_{k}=u_{k}$. Then, if (52) holds for all $x$, it follows that the distribution of refresh age $A_{D}(t)$ sampled in update points $u_{k}$ equals that sampled in uniformly random instances $Q_{T}$, which in turn is equivalent to our earlier formulation (21). While we have proven that the right side of (52) exists and equals a constant almost surely, existence of the left side or its equality to the integral is not guaranteed in general cases.

ASTA analysis focuses on the properties of points $\left\{t_{k}\right\}$ and their relationship to $X(t)$ that allow (52) to hold; however, this normally requires conditions that are difficult to verify in practice (e.g., LAA, WLAA, LBA [27]). We therefore discuss general guidelines for ensuring that (52) is satisfied, without becoming engrossed in unnecessary rigor.

Knowing that constant $D$ is optimal from the staleness perspective, we are now interested in the ability (or lack thereof) of $N_{D}$ to sample $N_{U}$ (and vice versa) in ways that achieve age-independence in (52), but without sacrificing optimality of $D$. One of the main concerns is that if all $U_{i}$ are constant, then it is possible that $N_{D}$ may sample update age that is different from that observable by a uniform $Q_{T}$. For example, suppose process $N_{U}$ has $U_{i}=5$ for all $i \geq 1$ and process $N_{D}$ has $D_{1}=$ uniform $[0,5]$ and $D_{k}=5$ for $k \geq 2$. Since the refresh process is stationary, the two ages $A_{D}\left(Q_{T}\right)$ and $A_{U}\left(Q_{T}\right)$ are independent random variables when considered across all sample paths, but the same conclusion and thus (52) fail to hold in any single execution of the system.

While sampling constant update cycles $\left\{U_{i}\right\}$ with constant synchronization intervals $\left\{D_{k}\right\}$ may seem like a bad idea, asymptotic age-independence is sometimes possible even in such cases. To build intuition, suppose $D_{k}=\pi$ for all $k \geq 1$. Then, from the equidistribution theorem, $A_{U}\left(d_{k}\right)=(k \pi)$ mod 5 is a uniformly random variable in $[0,5]$, meaning that $A_{U}\left(d_{k}\right)$ has the same distribution as $A_{U}\left(Q_{T}\right)$. The key observation is to ensure that $N_{D}$ puts its download points uniformly across the cycles of $N_{U}$.

In general, sequence $a_{k}=(k \xi) \bmod 1$, where $k=1,2, \ldots$ and $\xi$ is an irrational constant, is uniformly distributed in $[0,1]$, in which case for any Riemann-integrable function, an ASTA- like condition automatically holds:

$$
\lim _{n \rightarrow \infty} \frac{1}{n} \sum_{k=1}^{n} f\left(a_{k}\right)=\int_{0}^{1} f(x) d x .
$$

The integration limit can be extended if $f(x)$ is periodic. Assuming its period $\tau$ is not an integer multiple of $\xi$, sequence $b_{k}=(k \xi) \bmod \tau$ is uniform in $[0, \tau]$ and thus:

$$
\lim _{n \rightarrow \infty} \frac{1}{n} \sum_{k=1}^{n} f(k \xi)=\frac{1}{\tau} \int_{0}^{\tau} f(x) d x .
$$

While using $D_{k}=\pi$ to sample $U_{i}=5$ works well, there is a possibility that $U_{i}$ itself happens to be a multiple of $\pi$. To preclude these cases, $N_{D}$ must exhibit enough randomness to prevent $D_{k} / U_{i}$ from becoming deterministically an integer. This leads us to the next definition.

Definition 8: A distribution $F(x)$ of random variable $Y$ is called lattice if there exists a constant $c$ such that $Y / c$ is always an integer.

Non-lattice distributions may be entirely continuous, which includes the classical PASTA (Poisson Arrivals See Time Averages) [40] with exponential $D_{k}$ and the uniform distribution often suggested for network measurement [3]. However, they can also be entirely discrete. In such cases, cycle lengths of $F_{D}(x)$ must spread the probability mass across at least two values $(a, b)$, where $a / b$ is irrational, e.g., pairs $(\pi, 1)$ or $(e, \sqrt{2})$. By bringing spread $|b-a|$ closer to zero, it is possible to obtain a variety of approximations to the optimal (constant) synchronization delay with mean $l=(a+b) / 2$.

Theorem 11: In scenarios with iid $\left\{D_{k}\right\}$ and non-lattice $F_{D}(x)$, age-independence holds for all $N_{U}(x)$.

Proof: The result follows from the equidistribution theorem and the iid nature of $\left\{D_{k}\right\}$.

Conversely, in certain cases, it may be known a-priori that $N_{U}$ is non-lattice renewal. Then, it is not difficult to see that constant $D$ guarantees age-independence, which means that optimality is achievable in practice under these conditions.

\section{APPLICATIONS}

We now examine the presence of Poisson updates in real data sources and show how to apply the developed models to solve several classes of multi-source/replica problems.

\section{A. Real-Life Update Processes}

We first discuss possible reasons for the frequent use of memoryless source-update processes in the literature. If indeed this is universal, extensions to non-Poisson dynamics may be unnecessary. While modeling convenience is one possible explanation [11], there is certain belief in the field that updates to individual web pages can be accurately described by a Poisson process, which has fueled this line of modeling for over a decade [4], [5], [8], [9], [10], [15], [16], [17], [19], [22], [25], [26], [28], [29], [33], [35], [42].

Intuitively, there is no fundamental reason why a single source should exhibit Poisson dynamics, especially when modified by humans. A more likely scenario would be heavytailed behavior observed in many areas of computer networks 


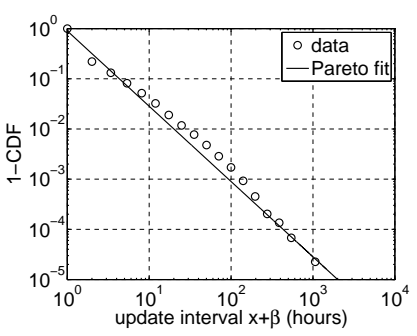

(a) distribution tail $\bar{F}_{U}(x)$

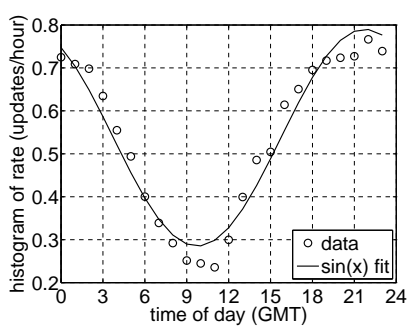

(c) update rate

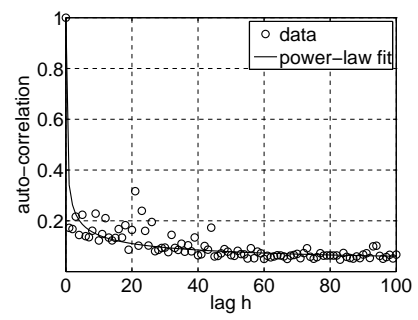

(b) correlation $\rho(h)$

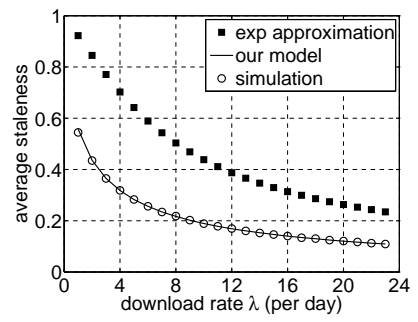

(d) model accuracy
Fig. 9. George W. Bush page dynamics.

[12], [23], [30] and user-driven distributed systems [6], [32], [34]. Another intuitively reasonable inter-update distribution is constant, where certain information is injected into the system periodically by design or is obtained from an ON/OFF source (e.g., sensors trying to conserve energy).

Closer examination of the origin [9] of the Poisson conclusion reveals several limitations. First, the distribution of page inter-update intervals was sampled using incomplete observation, meaning that some of the updates went unnoticed. As a result, bias could have been introduced in the measurements. Second, the exponential distribution was fitted to updates of multiple pages rather than a single page. Poisson dynamics have been known to emerge when aggregating arrival processes [1] and summing up variables [2], which does not tell us much about the individual distributions being combined. Finally, to conclude that $N_{U}$ is Poisson, it is insufficient to observe an exponential distribution in $\left\{U_{i}\right\}_{i=1}^{\infty}$; instead, one must also show stationary independent increments [41].

\section{B. Wikipedia}

Many of these pitfalls can be avoided if model verification is performed over sources that expose information about each update. One particularly interesting source with public traces of all modification timestamps is Wikipedia [37]. From a search-engine perspective, this website represents a realistic example of data churn stemming from user interaction with each other (e.g., edits from other people), flash crowds in response to external events, and diurnal activity patterns of the human lifecycle. Wikipedia is also well-suited for purposes of model validation and discussion.

To shed light on the complexity of real $F_{U}(x)$, we plot in Fig. 9(a) the tail CDF of inter-update delay for the most frequently modified article - "George W. Bush" with 44, 296 updates in 10 years (mean delay $E[U]=1.86$ hours). The figure is a close match to Pareto tail $(1+x / \beta)^{-\alpha}$ with $\alpha=1.4$ and $\beta=0.93$. In Fig. 9(b), we show the corresponding auto- correlation function $\rho(h)$ with a power-law fit $h^{-0.37}$, which suggests long-range dependence (LRD) with Hurst parameter 0.81. Of course, LRD effects might be caused and/or compounded by non-stationarity. To address this question, Fig. 9(c) shows the update rate throughout the day, clearly indicating non-stationary dynamics.

This example underscores the need to keep the model general and not limit results to renewal or even stationary cases, which was our goal with assumptions (5)-(6). Approximating $F_{U}(x)$ as non-lattice and using constant $D$, we next compute the probability of staleness for this page by supplying (31) with George W. Bush' empirically computed distribution $F_{U}(x)$. We contrast the result against the closest Poisson formula $1-\lambda\left(1-e^{-\mu / \lambda}\right) / \mu$ from [9]. Fig. 9(d) shows that (31) is accurate, but the Poisson approximation suffers over $100 \%$ relative error for much of the examined range.

What is more important is the performance of the model in providing an accurate assessment of the download bandwidth needed to achieve a given $p$. We invert the formulas to solve for $\lambda$ as a function of $p$ and plot the result in Fig. 10(a). These results show a much more dramatic difference. For example, $20 \%$ staleness requires 95 downloads/day according to previous Poisson models, while in reality this can be achieved with just 8 . To illustrate this better, we show the ratio of these two curves in Fig. 10(b), where the amount of Poisson overestimation varies from one to almost two orders of magnitude depending on the desired $p$.

\section{Aggregation (Many-to-One)}

When a single replica tracks $M$ sources, as in Fig. 1(a), performance is assessed by its ability to provide usable aggregate information to the consumer. If sources are independent, many results are relatively easy to obtain. For example, consider a system that selects a particular replica and loads it with a MapReduce job that has to execute over the data of all sources. A computation may be considered successful if at least one source is fresh at the time of job request. Then, the fraction of successful attempts is $1-\prod_{i=1}^{M} p_{i}$, where $p_{i}$ in (25) is the probability of staleness for source $i$. Alternatively, application consistency may require that all sources be simultaneously fresh, which leads to the probability of success via $\prod_{i=1}^{M}\left(1-p_{i}\right)$.

A more interesting problem is optimal allocation of download rates to different sources. Suppose $q_{i}$ is the probability that an incoming query requests data from source $i$ and $\mu_{i}$ is its update rate. Then, the goal is to allocate refresh rates $\lambda_{i}$ so as to optimize the expected staleness cost $C\left(\lambda_{i}, \mu_{i}\right)$ for a given bandwidth budget $\Lambda$ :

$$
\min \sum_{i=1}^{M} q_{i} C\left(\lambda_{i}, \mu_{i}\right) \quad \text { subject to } \quad \sum_{i=1}^{M} \lambda_{i} \leq \Lambda,
$$

where $C\left(\lambda_{i}, \mu_{i}\right)$ refers to either $\bar{\eta}$ or $\bar{\rho}$.

For $\Lambda \ll \sum_{i=1}^{M} \mu_{i}$ and certain choices of $w(x)$, solutions to (55) using cost $\bar{\eta}$ are known to completely starve frequently modified sources in favor of those that are updating slowly [9]. Since (55) does not have a closed-form solution under $\bar{\eta}$ even in the simplest cases, specific conditions for starvation 


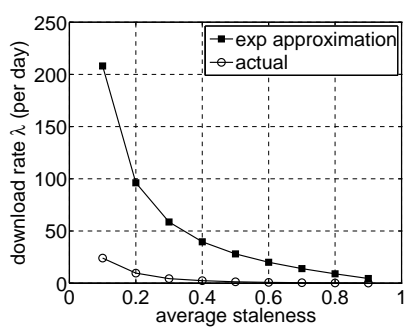

(a) crawl rate

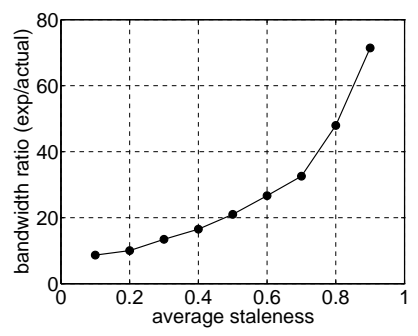

(b) ratio
Fig. 10. Application of staleness models to the update process of George W. Bush.

are not clear. Complete loss of synchronization for sources whose $\mu_{i}$ is above some (typically unknown) threshold may be an unwelcome surprise for many applications. This naturally leads to the question of whether $\bar{\rho}$ suffers from the same drawback. We address this next.

Theorem 12: Assume $q_{i} \mu_{i}>q_{j} \mu_{j}>0$ and let refresh delays be optimal (i.e., constant). Then, the solution to (55) using $\bar{\rho}$ guarantees that $\lambda_{i}>\lambda_{j}>0$.

Proof: Using Lagrange multipliers, we get that all partial derivatives of $q_{i} C\left(\mu_{i}, \lambda_{i}\right)$ must equal some constant $\kappa$ :

$$
\kappa=-\frac{\partial\left[q_{i} C\left(\mu_{i}, \lambda_{i}\right)\right]}{\partial \lambda_{i}}=-q_{i} \mu_{i} \frac{\partial\left[\lambda_{i} w_{3}\left(1 / \lambda_{i}\right)\right]}{\partial \lambda_{i}},
$$

which follows from (39) and $D_{i}=1 / \lambda_{i}$. Expanding, we get $\kappa=q_{i} \mu_{i} f\left(\lambda_{i}\right)$, where

$$
f(x)=\frac{w_{2}(1 / x)}{x}-w_{3}(1 / x)
$$

is a monotonically non-increasing function:

$$
f^{\prime}(x)=-\frac{w_{2}(1 / x)}{x^{2}}-\frac{w(1 / x)}{x^{3}}+\frac{w_{2}(1 / x)}{x^{2}}=-\frac{w(1 / x)}{x^{3}} .
$$

Notice that $f\left(\lambda_{i}\right) \geq 0$ for all $\lambda_{i}$ since $w_{3}$ is an integral of $w_{2}(x)$ from 0 to $1 / \lambda_{i}$. Therefore, $\kappa \geq 0$ and the relationship between $\mu_{i}$ and $\lambda_{i}$ is determined by:

$$
\lambda_{i}=f^{-1}\left(\frac{\kappa}{q_{i} \mu_{i}}\right) .
$$

If since $f$ is non-increasing, larger $q_{i} \mu_{i}$ implies larger $\lambda_{i}$. Finally, since $f(x)>0$ for all $x>0$, it follows that its inverse $f^{-1}$ has the same properly and thus no positive $q_{i} \mu_{i}>0$ can achieve $\lambda_{i}=0$. This means the optimal allocated rate must be strictly positive (i.e., no starvation).

To explain how optimization with $\bar{\rho}$ can be used, we assume constant $D$ and $w(x)=1$, with the goal to maximize $\sum_{i=1}^{M} q_{i} E\left[M_{i}(t)\right]$. Solving (55), the optimal download rate of each page is proportional to the square root of $q_{i} \mu_{i}$ :

$$
\lambda_{i}=\Lambda \frac{\sqrt{q_{i} \mu_{i}}}{\sum_{j=1}^{M} \sqrt{q_{j} \mu_{j}}} .
$$

The optimal penalty is then:

$$
\sum_{i=1}^{M} q_{i} E\left[M_{i}(t)\right]=\sum_{i=1}^{M} \frac{q_{i} \mu_{i}}{2 \lambda_{i}}=\frac{\left(\sum_{j=1}^{M} \sqrt{q_{j} \mu_{j}}\right)^{2}}{2 \Lambda} .
$$

Define random variable $\mu$ to have the same distribution as $\left\{\mu_{1}, \ldots, \mu_{M}\right\}$. Then, for the most basic scenario where all pages are equally popular, i.e., $q_{i}=1 / M$, we get:

$$
\sum_{i=1}^{M} q_{i} E\left[M_{i}(t)\right]=M \frac{E[\sqrt{\mu}]^{2}}{2 \Lambda} .
$$

For the other extreme, where pages are searched for in proportion to their modification rate, i.e., $q_{i} \sim \mu_{i}$, we have:

$$
\sum_{i=1}^{M} q_{i} E\left[M_{i}(t)\right]=M \frac{E[\mu]}{2 \Lambda} .
$$

To put these models in perspective, we use Wikipedia's distribution of $\mu$, which happens to be quite heavy-tailed (i.e., Zipf shape $\alpha=0.6$ ). The average update rate across all pages is $E[\mu]=8$ updates/day; however, $98 \%$ of them exhibit $\mu_{i}$ less than $1 /$ day, $90 \%$ less than $1 /$ week, and $50 \%$ below $8 /$ year. Using this distribution in (62) and (63) shows that optimizing staleness of the entire Wikipedia under uniform page access $q_{i}=1 / M$ requires 46 times less bandwidth $\Lambda$ than under Zipf. This can be explained by the fact that keeping frequently modified pages fresh costs more bandwidth. This effect is related to the variance of $\sqrt{\mu}$ :

$$
\frac{E[\mu]}{E[\sqrt{\mu}]^{2}}=\frac{\operatorname{Var}[\sqrt{\mu}]+E[\sqrt{\mu}]^{2}}{E[\sqrt{\mu}]^{2}}=\frac{\operatorname{Var}[\sqrt{\mu}]}{E[\sqrt{\mu}]^{2}}+1 .
$$

Consider extrapolating these results to $M=100 \mathrm{~B}$ sources and keeping the expected consumer lag $\sum_{i=1}^{M} q_{i} E\left[M_{i}(t)\right]$ below $\omega$ updates. We use the two models above as lower/upper bounds on the actual search-engine crawl rate. The first case requires download capability $\Lambda_{1}=M \cdot E[\sqrt{\mu}]^{2} / 2 \omega=99 / \omega$ thousand pages per second (pps), while the second one $\Lambda_{2}=$ $M \cdot E[\mu] / 2 \omega=4.6 / \omega$ million pps. For $\omega=10$ and $25 \mathrm{~KB}$ per page, these translate into 2 and $92 \mathrm{Gbps}$, respectively. Results can be easily adjusted to non-Wikipedia situations as long as $E[\sqrt{\mu}]$ and $E[\mu]$ are known.

\section{Load-Balancing (One-to-Many)}

The issue of redundant replication from a single source, as in Fig. 1(b), to $m$ nodes is quite different from the opposite case considered in the previous subsection. When the source fails, suppose the goal is to deduce the expected penalty afforded by the freshest member of the entire collection of $m$ replicas. The issue at stake is how this $1 \times m$ scenario compares to a single replica with some refresh rate $\lambda$ and optimal $D$. To keep comparison fair, assume that each of the $m$ replicas is allowed budget $\lambda / m$ in synchronization with the source. Decentralized operation leads to much better robustness under failure, but is it possible that this causes reduced freshness? If so, what is the amount of extra download bandwidth needed to keep both scenarios equally stale?

The main caveat in solving this problem is that staleness at different replicas is no longer independent. This happens because updates at the source simultaneously make all copies outdated, which means that reliability does not benefit exponentially with increased $m$. To overcome this issue, let $N_{D}^{1}, \ldots, N_{D}^{m}$ be the download processes used by the individual replicas. Then, observe that the entire collection can be 
replaced by a single replica that implements a refresh pattern $N_{D}^{*}$, which is a superposition of all point processes $\left\{N_{D}^{i}\right\}_{i=1}^{m}$. Therefore, the source can be recovered during the crash with a probability determined solely by $N_{D}^{*}$.

If we assume centralized scheduling between the replicas, then it is possible to run the system optimally (i.e., using a perfectly spaced out round-robin) and thus keep the overall penalty exactly the same as with a single replica. Under fully decentralized (i.e., independent) replica operation and $m \rightarrow \infty$, each rate $\lambda / m \rightarrow 0$ and thus $N_{D}^{*}$ likely converges in distribution to a Poisson process with rate $\lambda$ (Palm-Khintchine theorem [18]). This creates a problem, however, because exponential $D$ requires noticeably more overhead than constant $D$ to achieve the same staleness penalty. For example, using our model for $\bar{\rho}$ and discussion after (43), this difference is by a factor of 2 for $w(x)=1$ and by a factor of 6 for $w(x)=x$, which shows that a distributed cluster of replicas may need to consume $100-500 \%$ more bandwidth than a centralized solution for a given level of QoS (quality-of-service).

\section{E. Many-to-Many}

We conclude the paper by noting that Internet applications often combine the last two scenarios, i.e., $M \times 1$ and $1 \times m$ replication, into a single framework. However, these problems are usually separable into subproblems that can be reduced to the analysis above. For example, suppose we are interested in the probability that a query to a random subset of $j$ replicas finds at least one of the $k$ sources fresh. First, we compute the staleness probability for each source based on the aggregate synchronization process $N_{D}^{*}$ from $j$ replicas. Second, since each source is independent, we multiply these probabilities to deduce the likelihood that all $k$ sources are stale. Taking the complement of the result, we get the desired probability.

\section{CONCLUSION}

The paper introduced a novel model of sampled age under general non-Poisson update/synchronization processes and applied it to obtain many useful metrics of staleness. We additionally established that constant inter-refresh intervals were optimal for all considered cases and provided guidelines for achieving ASTA even in those cases. We finally considered a family of related problems stemming from $1 \times m$ and $M \times 1$ replication, showing that they can be easily solved from the preceding analysis of the $1 \times 1$ case.

Future work involves reducing staleness when $N_{D}$ is allowed to depend on observations of $N_{U}$ and/or prior knowledge of its distribution of update cycles $F_{U}(x)$.

\section{REFERENCES}

[1] S. L. Albin, "On Poisson Approximations for Superposition Arrival Processes in Queues," Management Sci., vol. 28, no. 2, pp. 126-137, 1982.

[2] R. Arratia, L. Goldstein, and L. Gordon, "Two Moments Suffice for Poisson Approximations: The Chen-Stein Method," The Annals of Probability, vol. 17, no. 1, pp. 9-25, Jan. 1989.

[3] F. Baccelli, S. Machiraju, D. Veitch, and J. Bolot, "The Role of PASTA in Network Measurement," in Proc. ACM SIGCOMM, Sep. 2006.

[4] B. E. Brewington and G. Cybenko, "How Dynamic is the Web," Computer Networks, no. 1-6, pp. 257-276, Jun. 2000
[5] L. Bright, A. Gal, and L. Raschid, "Adaptive Pull-based Policies for Wide Area Data Delivery," ACM Trans. Database Syst., no. 2, pp. 631671, June 2006.

[6] F. E. Bustamante and Y. Qiao, "Friendships that Last: Peer Lifespan and its Role in P2P Protocols," in Proc. Web Content Caching and Distribution, Sep. 2003.

[7] D. Carney, S. Lee, and S. Zdonik, "Scalable Application-Aware Data Freshening," in Proc. IEEE ICDE, March 2003, pp. 481-492.

[8] J. Cho and H. Garcia-Molina, "The Evolution of the Web and Implications for an Incremental Crawler," in Proc. VLDB, Sep. 2000, pp. 200-209.

[9] J. Cho and H. Garcia-molina, "Synchronizing a Database to Improve Freshness," in Proc. ACM SIGMOD, May 2000, pp. 117-128.

[10] J. Cho and H. Garcia-Molina, "Estimating frequency of change," $A C M$ Trans. Internet Technol., vol. 3, pp. 256-290, August 2003.

[11] E. G. Coffman, Z. Liu, and R. R. Weber, "Optimal Robot Scheduling for Web Search Engines," Journal of Scheduling, no. 1, pp. 15-29, Jun. 1998.

[12] M. E. Crovella and A. Bestavros, "Self-Similarity in World Wide Web Traffic: Evidence and Possible Causes," IEEE/ACM Trans. Netw., vol. 5, no. 6, pp. 835-846, 1997

[13] J. Dean and S. Ghemawat, "MapReduce: Simplified Data Processing on Large Clusters," in Proc. USENIX OSDI, Dec. 2004, pp. 137-150.

[14] D. Denev, A. Mazeika, M. Spaniol, and G. Weikum, "SHARC: Framework for Quality-Conscious Web Archiving," in Proc. VLDB, Aug. 2009.

[15] D. Dey, Z. Zhang, and P. De, "Optimal Synchronization Policies for Data Warehouses," INFORMS J. on Computing, no. 2, pp. 229-242, Jan. 2006.

[16] J. Eckstein, A. Gal, and S. Reiner, "Monitoring an Information Source Under a Politeness Constraint," INFORMS J. on Computing, no. 1, pp. 3-20, Jan. 2008.

[17] A. Gal and J. Eckstein, "Managing Periodically Updated Data in Relational Databases: a Stochastic Modeling Approach," J. ACM, no. 6, pp. 1141-1183, Nov. 2001.

[18] D. Heyman and M. Sobel, Stochastic Models in Operations Research, Volume 1. McGraw-Hill, 1982.

[19] Y. Huang, R. H. Sloan, and O. Wolfson, "Divergence Caching in ClientServer Architectures," in Proc. IEEE PDIS, Sep. 1994, pp. 131-139.

[20] Internet Archive. [Online]. Available: http://archive.org/.

[21] R. Ladin, B. Liskov, L. Shrira, and S. Ghemawat, "Providing High Availability Using Lazy Replication," ACM Trans. Comput. Syst., no. 4, pp. 360-391, Nov. 1992.

[22] J.-J. Lee, K.-Y. Whang, B. S. Lee, and J.-W. Chang, "An Update-Risk Based Approach to TTL Estimation in Web Caching," in Proc. WISE, Dec. 2002, pp. 21-29.

[23] W. E. Leland, M. S. Taqqu, W. Willinger, and D. V. Wilson, "On the Self-Similar Nature of Ethernet Traffic,"' in Proc. ACM SIGCOMM, Sep. 1993, pp. 183-193.

[24] Y. Ling and W. Chen, "Measuring Cache Freshness by Additive Age," SIGOPS Oper. Syst. Rev., vol. 38, pp. 12-17, Jul. 2004.

[25] Y. Ling and J. Mi, "An Optimal Trade-off between Content Freshness and Refresh Cost," Applied Probability, vol. 41, no. 3, pp. 721-734, Sep. 2004.

[26] N. Matloff, "Estimation of Internet File-access/Modification Rates from Indirect Data," ACM Trans. Model. Comput. Simul., vol. 15, pp. 233253, Jul. 2005.

[27] B. Melamed and W. Whitt, "On Arrivals That See Time Averages," Operations Research, vol. 38, no. 1, pp. 156-172, 1990.

[28] C. Olston and J. Widom, "Best-Effort Cache Synchronization With Source Cooperation," in Proc. ACM SIGMOD, May 2002, pp. 73-84.

[29] C. Olston and S. Pandey, "Recrawl Scheduling Based on Information Longevity," in Proc. WWW, Apr. 2008, pp. 437-446.

[30] K. Park, G. Kim, and M. Crovella, "On the Relationship Between File Sizes, Transport Protocols, and Self-Similar Network Traffic," in Proc. IEEE ICNP, Oct. 1996, pp. 171-180.

[31] S. Resnick, A Probability Path. Boston, MA: Birkhäuser, 1999.

[32] S. Saroiu, P. K. Gummadi, and S. D. Gribble, "A Measurement Study of Peer-to-Peer File Sharing Systems," in Proc. SPIE/ACM Multimedia Computing and Networking, vol. 4673, Jan. 2002, pp. 156-170.

[33] K. C. Sia and J. Cho, "Efficient Monitoring Algorithm for Fast News Alerts," IEEE Trans. Knowledge and Data Engineering, no. 7, pp. 950961, Jul. 2007.

[34] D. Stutzbach and R. Rejaie, "Understanding Churn in Peer-to-Peer Networks," in Proc. ACM IMC, Oct. 2006, pp. 189-202.

[35] Q. Tan and P. Mitra, "Clustering-based Incremental Web Crawling," ACM Transactions on Information Systems, no. 4, pp. 1-27, Nov. 2010. 
[36] B. Urgaonkar, A. G. Ninan, M. Salimullah, R. Shenoy, and K. Ramamritham, "Maintaining Mutual Consistency for Cached Web Objects," in Proc. IEEE ICDCS, Apr. 2001, pp. 371-380.

[37] Wikipedia Dumps. [Online]. Available: http://dumps.wikimedia.org/ enwiki/20110317/.

[38] G. Wise and E. Hall, Counterexamples in Probability and Real Analysis. Oxford Univ. Press, 1993.

[39] J. L. Wolf, M. S. Squillante, P. S. Yu, J. Sethuraman, and L. Ozsen, "Optimal Crawling Strategies for Web Search Engines," in Proc. WWW, May 2002, pp. 136-147.

[40] R. W. Wolff, "Poisson Arrivals See Time Averages," Operations Re- search, vol. 30, no. 2, pp. 223-231, 1982.

[41] R. W. Wolff, Stochastic Modeling and the Theory of Queues. Prentice Hall, 1989.

[42] M. Yang, H. Wang, L. Lim, and M. Wang, "Optimizing Content Freshness of Relations Extracted From the Web Using Keyword Search," in Proc. ACM SIGMOD, June 2010, pp. 819-830.

[43] H. Yu and A. Vahdat, "Design and Evaluation of a Continuous Consistency Model for Replicated Services," in Proc. USENIX OSDI, June 2000, pp. 305-318. 